\title{
SoundHunters: Increasing Learner Phonological Awareness in Plains Cree
}

\author{
Delaney Lothian, Gokce Akcayir $(\mathbb{0}$, Anaka Sparrow, Owen Mcleod $(1)$ \\ and Carrie Demmans Epp ${ }^{(\varpi)}$ \\ EdTeKLA Research Group, University of Alberta, Edmonton, AB, Canada \\ \{dlothian, akcayir, asparrow, omcleod, cdemmansepp\} @ualberta.ca
}

\begin{abstract}
Indigenous languages have been dying out due to colonial practices that limited and even punished their use. For this reason, there is a need to support the maintenance and revitalization of these languages as part of the reconciliation process. However, there has been little research to guide the use of technology in supporting language revitalization. To contribute to this process, this study investigated the use of a novel e-learning activity for a specific Indigenous language Plains Cree (nehiyawewin). This activity, SoundHunters, targets the development of learner phonological awareness (i.e., their ability to understand and manipulate sounds in a language) through game play. A mixed-methods study was used to measure learning and explore learner experiences. Learner performance on a transcription task, which required the mapping of sounds to characters, improved following SoundHunters use. The nature of learner errors indicates the development of learners' interlanguage and provides evidence of transfer from English to Cree. Additionally, learners enjoyed the activity while finding it appropriately challenging. These results show the potential for using adaptive technology to support learning in low-resource settings, such as those that exist for most Indigenous languages.
\end{abstract}

Keywords: Computer assisted language learning · Indigenous languages ·

Listening skills

\section{Introduction}

Canada wants to reconcile its previous actions against Indigenous peoples in Canada who have historically been mistreated and oppressed. In 2015, the Truth and Reconciliation Commission of Canada (TRC) released a report calling for the government to take action towards reconciliation [49]. One of the calls to action was the revitalization of the many Indigenous languages and cultures within Canada.

Like with learning any language, learning Indigenous languages includes the acquisition of reading, writing, speaking, and listening skills. Within these, excelling in listening

The original version of this chapter was revised: The reference numbers 28 and 34 have been updated. The correction to this chapter is available at https://doi.org/10.1007/978-3-030-52237-7_50 
and speaking requires phonological awareness which is knowledge of how sounds are used within a language [5]. This awareness gradually develops throughout childhood for one's first language, but it needs to be nurtured when learning additional languages [2]. Phonological awareness facilitates learners' speaking and listening skills. Supporting the development of these skills is of primary importance because of the oral traditions that are common amongst Indigenous cultures in Canada. The need for technologies to support this type of learning is pronounced because of the limited access learners have to instructors or speakers and the prevalence of dictionary-like vocabulary tools that fail to meet this community-identified need [35]. The current lack of ingenuity and adaptivity in the language-learning technologies that are available to learners of Indigenous languages [35] means that there has been little research investigating their effectiveness. Given this lack of investigation, we do not know which approaches support the development of the skills necessary for engaging in the learning practices that are of cultural value to specific Indigenous communities.

One of the approaches that might help learners develop core skills is game-based learning which has been garnering continued attention $[10,56]$. Its affordances such as providing immersive exposure to the language and decreasing anxiety [26], as well as creating an entertaining and interactive learning experience have contributed to its use within language learning. These are important factors in language learning because learners' willingness to use a language can foster improvement [1].

In addition to supporting language learning, game-based learning can trigger intrinsic motivation in learners [32] which might help them overcome the barriers that they face during learning. This additional motivation is especially important to Indigenous language learning as there are many social, technical, and access barriers to acquiring these languages $[21,35,37]$. The popularity of Duolingo has demonstrated technology's ability to provide a captivating language learning experience [24]. For these reasons, a gamebased e-learning activity that aims to develop learner phonological awareness could support Indigenous language learning as learner knowledge of phonology is predictive of several other language learning tasks [52].

\section{Related Literature}

Language learning has a long history of using games and simulations in both digital and non-digital learning contexts [12]. Most studies of technology used to support language learning have focused on enhancing the vocabulary knowledge of learners [9, 17, 54], with only a handful of systems using adaptive features to support oral language acquisition. For example, VocabNomad was a mobile communication support tool that enabled listening and pronunciation practice with recommendations being used to expose learners to new vocabulary items [16, 18]. Many other systems focus on supporting grammar instruction [9]. Among the systems and approaches that support learner's grammatical knowledge and writing skills are Grammarly [38] and the use of machine learning approaches for detecting or correcting grammatical errors [48]. When investigating the combined impact of feedback with such approaches, the addition of self-explanation within an adaptive grammar tutor failed to improve retention over practice alone [55]. Other than feedback and practice, a learner's writing abilities benefit from improved reading proficiency as increased receptive knowledge is predictive of productive knowledge [53]. Keeping this in mind, a system that was designed to support English language 
learners when they are trying to read authentic texts [57] might benefit both skills. However, the effectiveness of this system has yet to be evaluated. As summarized in reviews [45] and demonstrated by the above explorations and system designs, most adaptive computer-assisted language learning applications have focused on receptive skills (e.g., grammar, reading) or text-based approaches. This may be partly due to the technological and other challenges associated with supporting oral and productive language learning activities [31].

While less studied [9, 17], investigations examining how to improve language learners' speaking and listening have used a variety of technologies. One investigation showed that the most common learning activities performed by migrant language learners involved the repurposing of existing technologies to support pronunciation modelling and self-testing [17]. These types of interactions are dependent upon several features that include the ability to record, play audio or produce language through speech synthesis, and automatically analyze learner speech. One commercial technology that aimed to promote the identification and production of sounds used speech recognition to provide feedback on English language learners' pronunciation of single words [47]. Similarly, a game-based adaptive pronunciation tutor provided feedback on learner pronunciation [19]. This tutor was well received by language learners even though it was not shown to improve their phonemic awareness or pronunciation. In contrast, findings from a study where a mobile game was used to promote speaking and listening skills suggested the game only supported the improvement of learners' speaking abilities [28]. In contrast, a popular commercial language-learning application that includes activities which are expected to support the development of phonological awareness (i.e., Duolingo) was associated with improved listening skills [44], and an experimental system, called ToneWars, helped learners develop their declarative knowledge of Chinese tones through collaboration and competition [20]. Going beyond the simpler interactions seen in the above systems, the investigation of one of the few simulation-based systems that effectively supports language learning revealed that those who performed well in foundational skill-based activities, such as phonemic awareness, also performed better in simulation tasks [29]. A more recent example that aims to support communication practice focuses on adding features to agents in a way that will increase learner willingness to communicate [1].

A scoping review on the use of games in language learning revealed that $94 \%$ of studies were focused on English as an additional language [26] even though other languages could benefit from these types of approaches. It has even been argued that this class of approaches might help revitalize endangered and threatened Indigenous languages [22]. However, this vision has yet to be realized. In one attempt to support Indigenous language revitalization, Parker and colleagues [39] designed a game to support learners of Blackfoot (an Indigenous language in North America). This game asked players to follow instructions in Blackfoot to achieve a set of sub-goals; however, its effectiveness was not evaluated with users. Another study introduced a computer-based language learning activity to families who want to learn Ojibwe (anishinaabemowin): an Indigenous language spoken in North America. This activity aimed to promote Ojibwe acquisition by incorporating language learning into family activities [25]. An adaptive system for the appropriate use of pronouns in Maori (an Indigenous language in New Zealand) showed similar performance gains as those obtained from standard tutorials [51]. So far, the sole adaptive system for supporting the acquisition of Plains Cree (nehiyawewin) grammar 
has only been evaluated from the perspective of people's opinion about the system's design [6].

As can be seen in the literature, many technological approaches to supporting language learning can advance learner skills and knowledge. Although, some of them add little benefit over non-adaptive approaches (e.g., [55]). We have yet to see the evaluation of the effectiveness of adaptive or other language learning technologies for Indigenous languages. Given this information and a need for effective educational resources to support Indigenous language learning [34], we created a game that aims to support the development of phonological awareness in Plains Cree (nehiyawewin) with the hope that it would be the first step in a series that supports improved listening and speaking skills among learners.

\section{Method}

A mixed-methods approach was used where qualitative and quantitative data were triangulated [14]. This approach provided a holistic view of learners' experience and knowledge acquisition [50].

\subsection{Learning Technology: SoundHunters}

The approach under investigation was inspired by the arcade game called Space Invaders. Instead of defending against an alien invasion, the game asks learners to differentiate between sets of characters and identify the one that matches the sounds they are hearing. Note that there are two common ways that Cree is written, and within this study we will be focusing on the Standard Roman Orthography (SRO) writing system which is composed of eight consonants, two semi-vowels, and seven vowels.

The temporal pressure aspect of the game mechanics was used to help support the development of student fluency [42]. When the game starts, the user sees two to four deer move down the screen (Fig. 1). Each deer has text attached at its side. The learner is then given a sound that corresponds to one of the deer's text (i.e., the correct answer). This sound repeats until the learner identifies and shoots the correct deer.

SoundHunters has four tasks. The first tests the user's knowledge of sound to single character mappings, which is the base unit. The second tests their knowledge of the mapping between sounds and character pairs on the way to developing an understanding of how sound-to-character mappings interact when they are grouped into larger units. The third task tests their ability to distinguish minimal pairs (i.e., two words that are identical except for one sound, such as sun and fun), which is known to support the development of phonological awareness. The fourth task tests the user's ability to identify the word that was said and is the most ecologically valid task as learners will typically have to understand full words when interacting with others. Each task has three difficulty levels: easy, medium, and hard.

The difficulty for the first two tasks is determined in the same way. In both, distractor types are defined based on their similarity to the audio and visual cues associated with the target item (i.e., correct answer). When providing examples, we also provide an international phonetic alphabet (IPA) pronunciation guide. An example of a distractor 


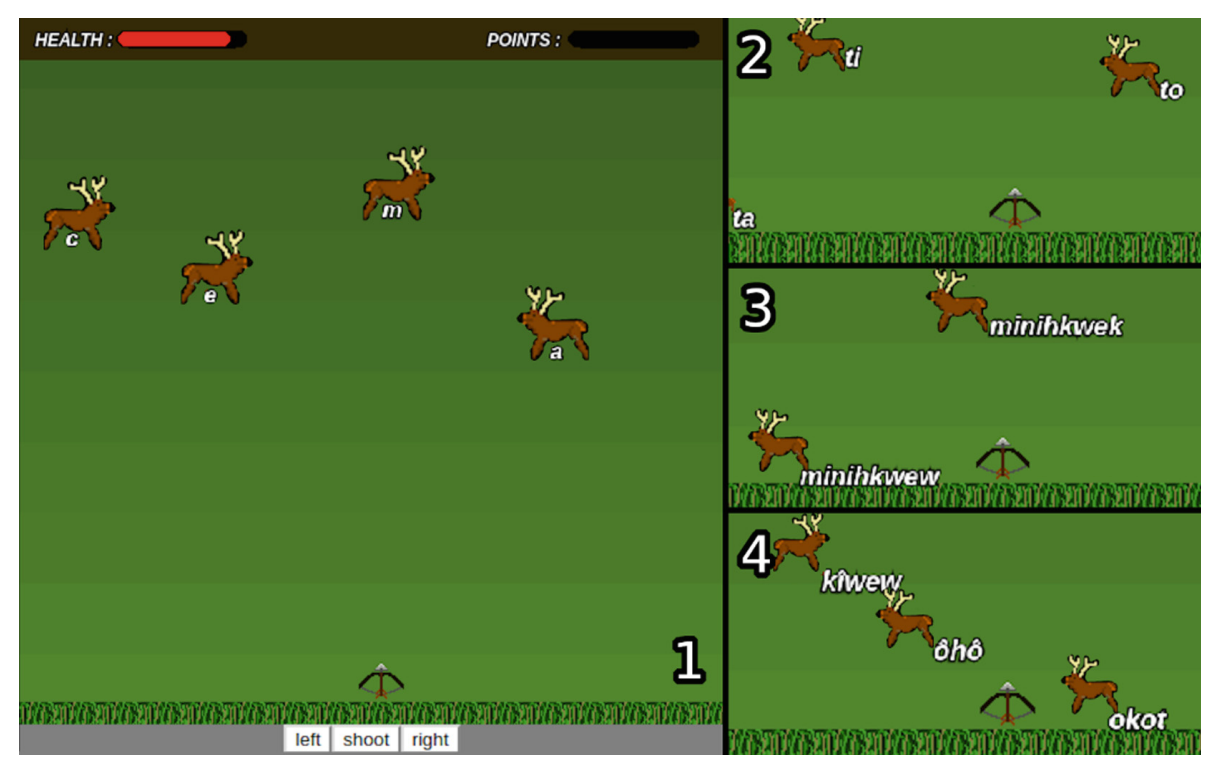

Fig. 1. Screenshots from game play. 1 shows the full screen for task 1 . Images 2 through 4 show a close up of the types of stimuli in tasks 2 through 4 respectively.

that includes aspects of both orthography and phonology would be "ô" (as in moose or $u$ in IPA) which is audibly and visually similar to o (as in put or $v$ in IPA). We refer to these types of distractors as bidimensional. A unidimensional distractor would be similar with respect to a single channel (i.e., audio or visual). For example, 'e' (as in bet or $\varepsilon$ in IPA) sounds similar to 'â' (as in fa or father - $a$ in IPA) but is not visually similar. A simple distractor would be a distractor that is neither visually nor audibly similar. For example, ' $\mathrm{t}$ ' is neither audibly nor visually similar to ' $a$ ' (as in cut or ə in IPA).

For both tasks one and two, the user is presented with two simple distractors and the correct answer at the easy level. At the medium difficulty level, the user is presented with a combination of three simple or unidimensional distractors and the correct answer. At the hard level, the user is presented with a combination of three unidimensional or bidimensional distractors and the correct answer. At the hard level, the speed at which the distractors descend also increases.

The nature of the third task limits the number of potential distractors to one. Consequently, the difficulty of the distractor in this task is based on its length and increased speed for the hard level.

The fourth task requires the user to identify the difference between words. Distractors were randomly chosen. Like task three, the difficulty was determined by the length of the word and the speed at which items descend. The speed increased for the medium level and again for the hard level. At the easy level, the user was presented with the correct answer plus two distractors. At the medium and hard level, they were presented with the correct answer plus three distractors. 


\subsection{Participants}

Participants were recruited via advertisements that included social media posts, posters around campus, and classroom announcements. Participant ages ranged from 19 to 76 $(\mathrm{M}=25.9, \mathrm{SD}=11.68) ; 2$ did not provide their age. Of the 25 participants, 13 were female, 11 male, and 1 participant opted not to specify. All were native English speakers who do not know Plains Cree. However, 4 said they can identify Plains Cree when they hear it. Participants were assigned a numeric identifier. This identifier (e.g., P1) is used to indicate which participant a quote originated from.

\subsection{Data Collection}

This study and its methods were pre-registered (https://osf.io/xjy4v). As planned, we used two measures to evaluate both the effectiveness of SoundHunters and learners' experience. These measures consist of a Cree knowledge test (as pre-test and post-test) and a questionnaire to evaluate player experience.

As a knowledge test, we asked participants to transcribe two short recordings of someone speaking in Cree. Each recording contained approximately 5-10 words in a single sentence. Each recording was 3-5 s long and the participant was allowed to listen to it a maximum of 3 times without pausing. Different recordings were used for the preand post-test. Scoring was handled based on correctness of the letters. Each correct letter earned one point. A half point was given to letters that do not exist in Plains Cree when their English sound represents a valid sound in Cree. An example would be if the user wrote ' $\mathrm{g}$ ' in place of ' $\mathrm{k}$ '. The ' $\mathrm{k}$ ' sound in Cree often sounds like ' $\mathrm{g}$ ' in English, but ' $\mathrm{g}$ ' is not used in SRO. The percentage of accumulated scores according to the number of letters in the correct answer (maximum score) are reported.

User experience was evaluated using the Model for Evaluating Educational Games (MEEGA+). This instrument was developed to analyze educational games and was reported to have high reliability (Cronbach's $\alpha=0.928$ ) with its subscales established through factor analysis [40].

\subsection{Data Analysis}

For the knowledge test, the normalized learning gain of each player was calculated [23, 41]. Additionally, using a paired sample t-test, we determined whether performance on the transcription test improved following system use. This test was conducted after determining that data followed a normal distribution. Errors on the pre- and post-test were grouped based on how the participant responded. These errors were compared across tests to identify evidence of changes in participant knowledge of specific sound-to-character mappings.

The analysis of the qualitative data began with reviewing the data from the perspective of participants' learning experience [46]. After that, we conducted thematic analysis to reveal themes within their learning experiences [8]. This process started with identifying codes which are expressions that relate to participants' learning experience. Then, these codes were grouped around themes and data were reviewed to confirm these themes. This process was handled by a researcher who has a PhD in educational technology and 
is experienced in qualitative data analysis. All steps of the process were reviewed and confirmed by another researcher to increase reliability (as in [43]).

\section{Results}

\subsection{Increased Phonological Awareness}

A paired-samples t-test of the phonemic knowledge tests revealed a significant improvement in learner performance $(t(24)=-6.85, p<.001, d=1.37)$ from the pre-test $(M$ $=49.37, S D=8.56)$ to the post-test $(M=58.50, S D=8.74)$. Participants' normalized learning gain showed considerable improvement, $95 \% \mathrm{CI}=[0.12,0.23]$, given that participants interacted with the system for approximately $49 \min (M=49 \min .21 \mathrm{~s}, S D=$ 6 min. $2 \mathrm{~s}$ ).

In addition to measurable changes in their knowledge, participants' open-ended responses indicate they learned Cree words or sounds which implies increased phonological awareness of Cree: "As levels progressed, I found I had a better understanding of different sounds" (P16) and "I feel like you get more used to the letters/sounds as you advance" (P14).

An examination of the errors participants made on the pre- and post-tests, indicates many mistakes were consistent across tests. These mistakes included using "e" (as in bet or $\varepsilon$ in IPA) instead of "i" (as in sit or I in IPA), or "oo" instead of "ô" (as in moose or $\mathrm{u}$ in IPA). These mistakes reflect the differences in the sound-to-character mappings between English (participants' native language) and Plains Cree. Along with these persistent errors, there were some types of errors that were different across tests. In the pre-tests, learners did not use accents where they were needed, such as using $i$ and $o$ (as in put or $\mho$ in IPA) instead of $\hat{i}$ (as in feel or i in IPA) and ô, whereas accents were observed in the post tests. In some cases, this meant that learners were using accents where they were not needed (e.g., ê instead of e, as e never takes an accent). Similarly, some consonants such as "h" and "w" were absent from the pre-test (e.g., waya was written instead of wâhyaw pronounced wahyau in IPA). In contrast, users included these consonants both where they were needed (e.g., mahtesa pronounced məhtesə in IPA) and in other locations which demonstrated negative transfer of sound-to-character mappings from English to Cree. For example, chin was written instead of cin, which is the SRO representation that is pronounced tsin in IPA.

\subsection{Learner Experience}

Figure 2 shows the distribution of participant responses to the learning experience questionnaire. Overall, participants reported positive experiences and positive perceptions of their learning. Their perception that they learned Cree sounds is consistent with the gains seen from the pre-test to the post-test. The themes that emerged from participant comments on their learning experience included the perceived learning of Cree words, having a positive learning experience, and facilitating learner attention.

The questionnaire results indicate that participating learners were challenged, had fun, and were highly satisfied with their learning experiences. Their open-ended comments confirm this finding of the game facilitating a positive learning experience. As participants said, 


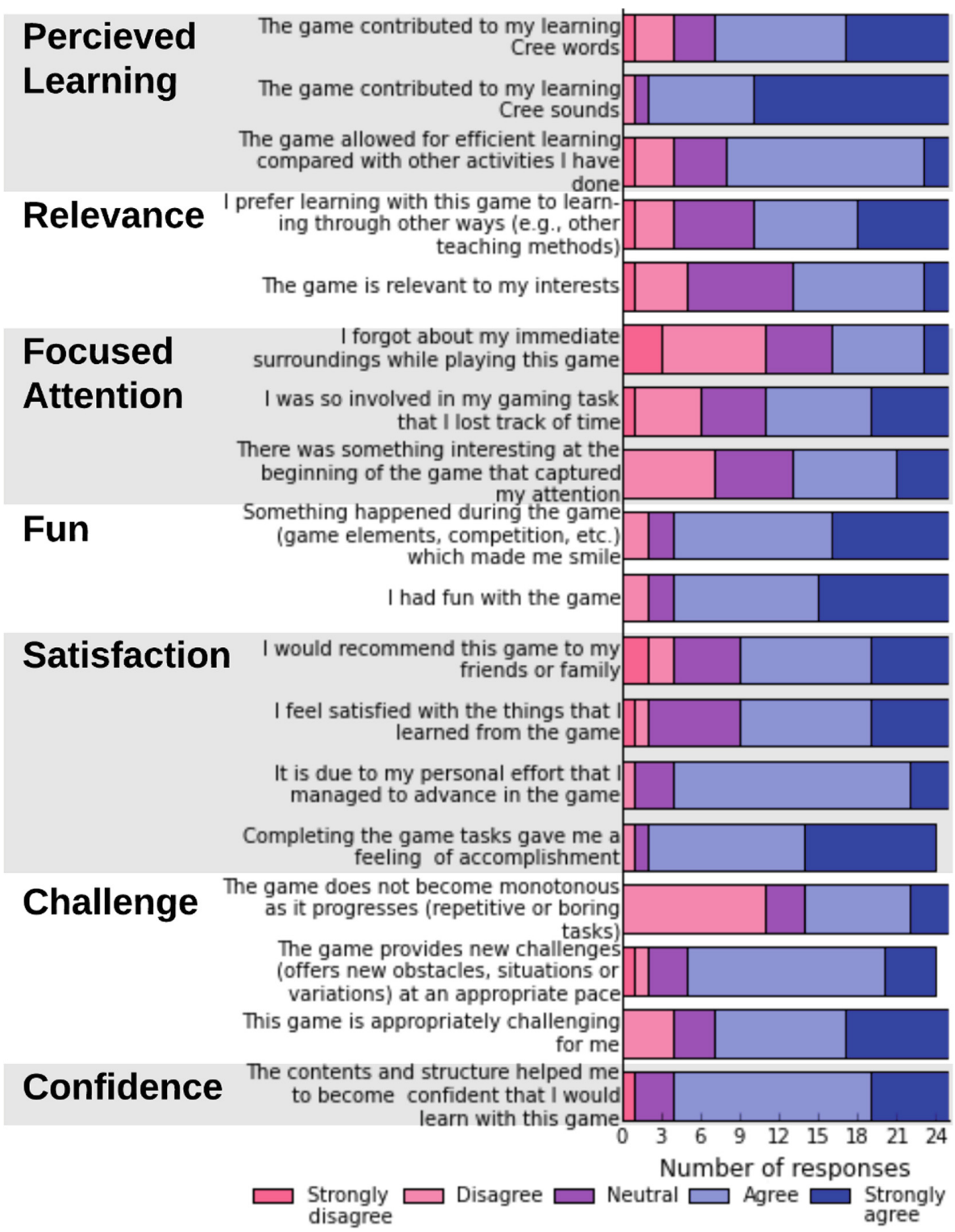

Fig. 2. Learners' play experience with SoundHunters

P9: "I really enjoyed the game. I thought it was a new and interesting way to help learn some basics on Cree word/sound structure. The game itself was simple enough that it didn't take away from learning but fun enough to keep interest". P17: "The game was a cool and easy way to learn Cree words/sounds. It was really useful to see both the spelling on screen and hear the sounds." 


\section{P13: "Finally hitting the correct target was very satisfying"}

Even though participants enjoyed learning in this way, their perceptions with respect to preferred learning approach did not necessarily indicate that using SoundHunters would be their first choice (Relevance, item 1, in Fig. 2). Their responses to the focused attention related items of the questionnaire indicate the game could have better captured their full attention even though their open-ended responses indicate that it helped them to focus on performing the learning task without making them feel like they were engaged in a learning activity: "It did grab my attention throughout playing the game" (P19) and it is a "learning tool where you can forget you're learning" (P12). One learner also stated that "it was simple enough and engaging to play" (P9), suggesting that its simplicity contributed to its capturing their attention.

\section{Discussion}

We explored the use of a game to improve phonological awareness for Indigenous language learning. The learning observed in this study provides empirical evidence for informal game-based learning practices, which generally lack strong support [7]. The observed improvement in participants' ability to map sounds to characters can be interpreted as a sign of the game's appropriate difficulty levels for the target audience since language-learning technologies that fail to provide the right amount of challenge are associated with a lack of learning [36].

Along with the amount of challenge, the type of interactivity provided could play a role in the activity's effectiveness, as some types of interactions that learners have with games have been associated with increased vocabulary retention [15]. In our game, the primary interaction with the user was through a combination of sounds and text which probably played an important role in the success of the game. These interactions are consistent with the learning objective of increasing learner phonemic awareness, with improvements shown through assessments that required learners to map sounds to characters, which is a fundamental component of phonemic awareness [4]. The repetition involved in the game is likely to have contributed to the measured improvements as repetition provides practice opportunities [13].

Learners' comments and questionnaire responses indicate they responded positively to this learning technology. More specifically, participants said they felt more like they were playing than studying, suggesting that the activity format eliminated the negative emotions, such as anxiety [27, 56], that are associated with foreign language learning as well as the learning of Indigenous languages [35]. As family-based approaches have helped improve Indigenous language learning [25] in other contexts, further game activities can be developed in a way that allows collaborative play so that families can engage with the learning activities together. This would additionally help to mitigate generational differences in Indigenous language learning.

Some of the errors participants made in the post-test indicated negative transfer, which is the expansion and use of existing knowledge from a well-known language to that of another language [3]. In this case, negative transfer was seen through the application of sound-to-character mappings from English during a Cree transcription task. To mitigate 
negative transfer, further activities can be planned to explicitly point out differences between these two languages [33]. The observed errors additionally provide evidence of the development of learners' interlanguage, which is a part of the language-learning process where learners combine characteristics of the first and additional languages [11] en route to eventual mastery of the target language if they persist.

\section{Conclusion}

In the last two decades, youth interest in learning their own Indigenous languages has been steadily increasing [21, 30]. However, these languages have few resources [34], research-informed learning activities [37], or personalized systems [6, 35] that learners can access to support their learning. Consistent with this, the need to develop technologies that support Indigenous language learning has been identified by community members [35]. To help meet this need, this study provided a novel instructional activity that promotes listening by developing learners' phonological awareness of Plains Cree using an adaptive game. Increases in learner knowledge as measured through a receptive task, demonstrate the potential for these types of activities, especially when considering how they enable access to learning that may not otherwise be possible, provide a safe practice environment where learners can afford to take risks, and transform the learning of an Indigenous language from something that was previously punishable to something that is enjoyable.

Acknowledgements. We acknowledge the support of the Natural Sciences and Engineering Research Council of Canada (NSERC) [RGPIN-2018-03834] and National Research Council Canada [contract 932832]. As part of our funding agreement, all code will be made publicly available through GitHub: https://github.com/EdTeKLA.

\section{References}

1. Ayedoun, E., Hayashi, Y., Seta, K.: L2 learners' preferences of dialogue agents: a key to achieve adaptive motivational support? In: Isotani, S., Millán, E., Ogan, A., Hastings, P., McLaren, B., Luckin, R. (eds.) AIED 2019. LNCS (LNAI), vol. 11626, pp. 19-23. Springer, Cham (2019). https://doi.org/10.1007/978-3-030-23207-8_4

2. Bae, H.S.: Metalinguistic awareness in vocabulary acquisition: evidence from grade six Korean EFL learners. Prim. Engl. Educ. 21(3), 5-22 (2015)

3. Bai, L., Qin, J.: A study of negative language transfer in college students' writing from cultural perspective. Theory Pract. Lang. Stud. 8(3), 306-313 (2018). https://doi.org/10.17507/tpls. 0803.05

4. Binder, K.S., Talwar, A., Bond, N.K., Cote, N.G.: From "Degisned" and "Dezine" to "Design". In: Perin, D. (ed.) The Wiley Handbook of Adult Literacy (2020). https://doi.org/10.1002/ 9781119261407.ch6

5. Blachman, B.A.: Phonological awareness. In: Kamil, M.L., Mosenthal, P.B., Pearson, P.D., Barr, R. (eds.) Handbook of Reading Research, pp. 483-502 Lawrence Erlbaum Associates Publishers, Mahwah (2016) 
6. Bontogon, M., Arppe, A., Antonsen, L., Thunder, D., Lachler, J.: Intelligent computer assisted language learning (ICALL) for nêhiyawêwin: an in-depth user-experience evaluation. Can. Mod. Lang. Rev. 74(3), 337-362 (2018). https://doi.org/10.3138/cmlr.4054

7. Boyle, E.A., et al.: An update to the systematic literature review of empirical evidence of the impacts and outcomes of computer games and serious games. Comput. Educ. 94, 178-192 (2016). https://doi.org/10.1016/j.compedu.2015.11.003

8. Braun, V., Clarke, V.: Using thematic analysis in psychology. Qual. Res. Psychol. 3(2), 77-101 (2006). https://doi.org/10.1191/1478088706qp063oa

9. Burston, J.: The reality of MALL: still on the fringes. CALICO J. 31(1), 103-125 (2014). https://doi.org/10.11139/cj.31.1.103-125

10. Chen, C.H., Liu, J.H., Shou, W.C.: How competition in a game-based science learning environment influences students' learning achievement, flow experience, and learning behavioral patterns. Educ. Technol. Soc. 21(2), 164-176 (2018). www.jstor.org/stable/26388392

11. Chen, H., Xu, H.: Quantitative linguistics approach to interlanguage development: a study based on the Guangwai-Lancaster Chinese Learner Corpus. Lingua 230, 1-15 (2019). https:// doi.org/10.1016/j.lingua.2019.102736

12. Connolly, T.M., Stansfield, M., Hainey, T.: An alternate reality game for language learning: ARGuing for multilingual motivation. Comput. Educ. 57(1), 1389-1415 (2011). https://doi. org/10.1016/j.compedu.2011.01.009

13. Coyne, R.: Mindless repetition: learning from computer games. Des. Stud. 24(3), 199-212 (2003). https://doi.org/10.1016/S0142-694X(02)00052-2

14. Creswell, J.W.: Research Design: Qualitative, Quantitative, and Mixed Methods Approaches. Sage Publications, Thousand Oaks (2014)

15. DeHaan, J., Reed, M.W., Kuwada, K.: The effect of interactivity with a music video game on second language vocabulary recall. Lang. Learn. Technol. 14(2), 74-94 (2010)

16. Demmans Epp, C., Tsourounis, S., Djordjevic, J., Baecker, R.M.: Interactive event: enabling vocabulary acquisition while providing mobile communication support. In: Lane, H.C., Yacef, K., Mostow, J., Pavlik, P. (eds.) AIED 2013. LNCS (LNAI), vol. 7926, pp. 932-933. Springer, Heidelberg (2013). https://doi.org/10.1007/978-3-642-39112-5_150

17. Demmans Epp, C.: Migrants and mobile technology use: gaps in the support provided by current tools. J. Interact. Media Educ. 1(2), 1-13 (2017). https://doi.org/10.5334/jime.432

18. Demmans Epp, C.: Mobile adaptive communication support for vocabulary acquisition. In: Lane, H.C., Yacef, K., Mostow, J., Pavlik, P. (eds.) AIED 2013. LNCS (LNAI), vol. 7926, pp. 876-879. Springer, Heidelberg (2013). https://doi.org/10.1007/978-3-642-39112-5_135

19. Demmans Epp, C., McCalla, G.: ProTutor: historic open learner models for pronunciation tutoring. In: Biswas, G., Bull, S., Kay, J., Mitrovic, A. (eds.) AIED 2011. LNCS (LNAI), vol. 6738, pp. 441-443. Springer, Heidelberg (2011). https://doi.org/10.1007/978-3-642-218699_63

20. Fan, X., Luo, W., Wang, J.: Mastery learning of second language through asynchronous modeling of native speakers in a collaborative mobile game. In: Proceedings of the CHI Conference on Human Factors in Computing Systems, pp. 4887-4898. ACM, New York (2017). https://doi.org/10.1145/3025453.3025544

21. Galla, C.K.: Indigenous language revitalization, promotion, and education: function of digital technology. Comput. Assist. Lang. Learn. 29(7), 1137-1151 (2016). https://doi.org/10.1080/ 09588221.2016.1166137

22. Galla, C.K.: Indigenous language revitalization and technology from traditional to contemporary domains. In: Reyhner, J., Lockard, L. (eds.) Indigenous Language Revitalization: Encouragement, Guidance \& Lessons Learned, pp. 167-182. Northern Arizona University, Flagstaff (2009) 
23. Hake, R.R.: Interactive-engagement versus traditional methods: a six-thousand-student survey of mechanics test data for introductory physics courses. Am. J. Phys. 66(1), 64-74 (1998). https://doi.org/10.1119/1.18809

24. Hampton, A.J., Nye, B.D., Pavlik, P.I., Swartout, W.R., Graesser, A.C., Gunderson, J.: Mitigating knowledge decay from instruction with voluntary use of an adaptive learning system. In: Penstein Rosé, C., et al. (eds.) AIED 2018. LNCS (LNAI), vol. 10948, pp. 119-133. Springer, Cham (2018). https://doi.org/10.1007/978-3-319-93846-2_23

25. Hermes, M., King, K.A.: Ojibwe language revitalization, multimedia technology, and family language learning. Lang. Learn. Technol. 17(1), 125-144 (2013). https://dx.doi.org/10125/ 24513

26. Hung, H.-T., Yang, J.C., Hwang, G., Chu, H., Wang, C.: A scoping review of research on digital game-based language learning. Comput. Educ. 126, 89-104 (2018). https://doi.org/ 10.1016/j.compedu.2018.07.001

27. Hwang, G.J., Hsu, T.-C., Lai, C.-L., Hsueh, C.-J.: Interaction of problem-based gaming and learning anxiety in language students' English listening performance and progressive behavioral patterns. Comput. Educ. 106, 26-42 (2017). https://doi.org/10.1016/j.compedu.2016. 11.010

28. Hwang, W.-Y., Shih, T.K., Ma, Z.-H., Shadiev, R., Chen, S.-Y.: Evaluating listening and speaking skills in a mobile game-based learning environment with situational contexts. Comput. Assist. Lang. Learn. 29(4), 639-657 (2016). https://doi.org/10.1080/09588221.2015.101 6438

29. Johnson, W.L., Wu, S.: Assessing aptitude for learning with a serious game for foreign language and culture. In: Woolf, B.P., Aïmeur, E., Nkambou, R., Lajoie, S. (eds.) ITS 2008. LNCS, vol. 5091, pp. 520-529. Springer, Heidelberg (2008). https://doi.org/10.1007/978-3540-69132-7_55

30. Krauss, M.: The condition of Native North American languages: the need for realistic assessment and action. Int. J. Soc. Lang. 132, 9-21 (1998). https://doi.org/10.1515/ijsl.1998. 132.9

31. Levy, M., Hubbard, P., Stockwell, G., Colpaert, J.: Research challenges in CALL. Comput. Assist. Lang. Learn. 28, 1-6 (2015). https://doi.org/10.1080/09588221.2014.987035

32. Lin, C.H., Huang, S., Shih, J., Covaci, A., Ghinea, G.: Game-based learning effectiveness and motivation study between competitive and cooperative modes. In: Proceedings of 17th International Conference on Advanced Learning Technologies (ICALT), pp. 123-127. IEEE, Timisoara (2017). https://doi.org/10.1109/ICALT.2017.34

33. Linck, J., Michael, E., Golonka, E., Twist, A., Schwieter, J.W.: Moving beyond two languages: the effects of multilingualism on language processing and language learning. In: Schwieter, J. (ed.) The Cambridge Handbook of Bilingual Processing, pp. 665-694 (2015). https://doi. org/10.1017/cbo9781107447257.030

34. Littell, P., Kazantseva, A., Kuhn, R., Pine, A., Arppe, A., Cox, C., Junker, M.-O.: Indigenous language technologies in Canada: assessment, challenges, and successes. In: Proceedings of the 27th International Conference on Computational Linguistics. pp. 2620-2632. Association for Computational Linguistics, Santa Fe, New Mexico, USA (2018). https://www.aclweb.org/ anthology/C18-1222/ 
35. Lothian, D., Akcayir, G., Demmans Epp, C.: Accommodating Indigenous people when using technology to learn their ancestral language. In: McCalla, F., Gutierrez, J., Olakanmi, O., Ishola, O. (eds.) International Workshop on Supporting Lifelong Learning co-located with the 20th International Conference on Artificial Intelligence in Education, vol. 2395, pp. 16-22 (2019). http://ceur-ws.org/Vol-2395/

36. Ma, Z.-H., Hwang, W., Chen, S., Ding, W.: Digital game-based after-school-assisted learning system in English. In: 2012 International Symposium on Intelligent Signal Processing and Communications Systems, pp. 130-135. IEEE, Taipei (2012). https://doi.org/10.1109/ISP ACS.2012.6473466

37. McIvor, O.: îkakwiy nîhiyawiyân : I am learning [to be] Cree. University of British Columbia (2012). https://doi.org/10.14288/1.0078368

38. ONeill, R., Russell, A.: Stop! Grammar time: university students' perceptions of the automated feedback program Grammarly. Australas. J. Educ. Technol. 35(1), 42-56 (2019). https://doi. org/10.14742/ajet.3795

39. Parker, J.R., Heavy Head, R., Becker, K.: Technical aspects of a system for teaching aboriginal languages using a game boy. In: Future Play, the International Conference on the Future of Game Design and Technology (2005)

40. Petri, G., Wangenheim, C.G., Borgatto, A.F.: MEEGA+, systematic model to evaluate educational games. In: Lee, N. (ed.) Encyclopedia of Computer Graphics and Games, pp. 1-7. Springer, Cham (2018). https://doi.org/10.1007/978-3-319-08234-9_214-1

41. Prather, E.E., Rudolph, A.L., Brissenden, G.: Teaching and learning astronomy in the 21st century. Phys. Today 62(10), 41-47 (2009). https://doi.org/10.1063/1.3248478

42. Presson, N., MacWhinney, B., Tokowicz, N.: Learning grammatical gender: The use of rules by novice learners. Appl. Psycholinguist. 35(4), 709-737 (2014). https://doi.org/10.1017/S01 42716412000550

43. Psaila, K., Fowler, C., Kruske, S., Schmied, V.: A qualitative study of innovations implemented to improve transition of care from maternity to child and family health $(\mathrm{CFH})$ services in Australia. Women Birth 27(4), e51-e60 (2014). https://doi.org/10.1016/j.wombi.2014.08.004

44. Putri, L.M., Islamiati, A.: Teaching listening using Duolingo application. Proj. (Prof. J. Engl. Educ.) 1(4), 460 (2018). https://doi.org/10.22460/project.v1i4.p460-465

45. Schulze, M., Heift, T.: Intelligent CALL. In: Thomas, M., et al. (eds.) Contemporary Computer-Assisted Language Learning, pp. 249-265. Bloomsbury, London (2012)

46. Strauss, A.L.: Qualitative Analysis for Social Scientists. Cambridge University Press, San Francisco (1987). https://doi.org/10.1017/CBO9780511557842

47. Tao, R.: Eyespeak. CALICO J. 25(1), 126-136 (2008)

48. Troussas, C., Chrysafiadi, K., Virvou, M.: Machine learning and fuzzy logic techniques for personalized tutoring of foreign languages. In: Penstein Rosé, C., et al. (eds.) AIED 2018. LNCS (LNAI), vol. 10948, pp. 358-362. Springer, Cham (2018). https://doi.org/10.1007/ 978-3-319-93846-2_67

49. Truth and Reconciliation Commission of Canada: Truth and reconciliation commission of Canada: Calls to action (2015). http://trc.ca/assets/pdf/Calls_to_Action_English2.pdf

50. Turner, S.F., Cardinal, L.B., Burton, R.M.: Research design for mixed methods: a triangulation-based framework and roadmap. Organ. Res. Methods 20(2), 243-267 (2017). https://doi.org/10.1177/1094428115610808

51. Vlugter, P., Knott, A., McDonald, J., Hall, C.: Dialogue-based CALL: a case study on teaching pronouns. Comput. Assist. Lang. Learn. 22(2), 115-131 (2009). https://doi.org/10.1080/095 88220902778260

52. Wade-Woolley, L., Geva, E.: Processing novel phonemic contrasts in the acquisition of L2 word reading. Sci. Stud. Read. 4(4), 295-311 (2000). https://doi.org/10.1207/S1532799X SSR0404_3 
53. Wagner, R.K., Muse, A.E., Tannenbaum, K.R.: Vocabulary Acquisition: Implications for Reading Comprehension. Guilford Press, New York (2007)

54. Wang, S., Wu, H., Kim, J.H., Andersen, E.: Adaptive learning material recommendation in online language education. In: Isotani, S., Millán, E., Ogan, A., Hastings, P., McLaren, B., Luckin, R. (eds.) AIED 2019. LNCS (LNAI), vol. 11626, pp. 298-302. Springer, Cham (2019). https://doi.org/10.1007/978-3-030-23207-8_55

55. Wylie, R., Sheng, M., Mitamura, T., Koedinger, K.R.: Effects of adaptive prompted selfexplanation on robust learning of second language grammar. In: Biswas, G., Bull, S., Kay, J., Mitrovic, A. (eds.) AIED 2011. LNCS (LNAI), vol. 6738, pp. 588-590. Springer, Heidelberg (2011). https://doi.org/10.1007/978-3-642-21869-9_110

56. Yang, Q.-F., Chang, S., Hwang, G., Zou, D.: Balancing cognitive complexity and gaming level: Effects of a cognitive complexity-based competition game on EFL students' English vocabulary learning performance, anxiety and behaviors. Comput. Educ. 148, 103808 (2020). https://doi.org/10.1016/j.compedu.2020.103808

57. Zilio, L., Fairon, C.: Adaptive system for language learning. In: 17 th International Conference on Advanced Learning Technologies (ICALT), pp. 47-49. IEEE, Timisoara (2017). https:// doi.org/10.1109/ICALT.2017.46 